\title{
Monte Carlo Sampling Method for a Class of Box-Constrained Stochastic Variational Inequality Problems
}

\author{
Pei-Yu Li iD \\ School of Mathematics, Southwest Minzu University, Chengdu 610041, China \\ Correspondence should be addressed to Pei-Yu Li; lipeiyu28@foxmail.com \\ Received 13 December 2020; Revised 9 February 2021; Accepted 27 April 2021; Published 2 June 2021 \\ Academic Editor: Hao Xiao \\ Copyright ( $\odot 2021$ Pei-Yu Li. This is an open access article distributed under the Creative Commons Attribution License, which \\ permits unrestricted use, distribution, and reproduction in any medium, provided the original work is properly cited. \\ This paper uses a merit function derived from the Fishcher-Burmeister function and formulates box-constrained stochastic \\ variational inequality problems as an optimization problem that minimizes this merit function. A sufficient condition for the \\ existence of a solution to the optimization problem is suggested. Finally, this paper proposes a Monte Carlo sampling method for \\ solving the problem. Under some moderate conditions, comprehensive convergence analysis is included as well.
}

\section{Introduction}

Let $l$ and $u$ be two $n$-dimensional vectors with components $l_{i} \in \mathbb{R} \cup\{-\infty\}$ and $u_{i} \in \mathbb{R} \cup\{+\infty\}$ satisfying $l_{i}<u_{i}$, and denote, by $S$, the nonempty and possibly infinite box $[l, u]:=\left\{x \in \mathbb{R}^{n} \mid l_{i} \leq x_{i} \leq u_{i}, i=1, \ldots, n\right\}$. Then, the boxconstrained variational inequality problem (BVIP, for short) is to find a vector $x^{*} \in S$ such that

$$
\left(x-x^{*}\right)^{T} F\left(x^{*}\right) \geq 0, \quad \forall x \in S,
$$

where $F: \mathbb{R}^{n} \longrightarrow \mathbb{R}^{n}$ is a given function. This problem is also called the mixed complementarity problem [1]. Both linear complementarity problems [2] and nonlinear complementarity problems [3] have played an important role in studying economic equilibrium and engineering problems $[4,5]$.

Much effort has been made to derive merit functions for the BVIP and then, using these functions, to develop solution methods. Recently, Kebaili and Benterki [6] propose a penalty approach for a box-constrained variational inequality problem (BVIP). It is replaced by a sequence of nonlinear equations containing a penalty term. A homotopy method for solving mathematical programs with box-constrained variational inequalities is presented [7]. A reformulation of the BVIP, based on the Fishcher-Burmeister function, is described in paper [8] by Sun and Womersley.
Further reformulations can be obtained by replacing the Fishcher-Burmeister function by the introduced function from [9] which seems to have somewhat stronger theoretical properties and a better numerical behavior.

Stochastic variational inequality problem (SVIP, for short) model is a natural extension of deterministic variational inequality models. Over the past few decades, deterministic variational inequality has been extensively studied for its extensive application in engineering, economics, game theory, and networks; see the book on the topic by Facchinei and Pang [1]. While many practical problems only involve deterministic data, there are some important instances where problem data contain some uncertain factors and consequently SVIP models are needed to reflect uncertainties. Gürkan et al. [10] have shown how to extend a simulation-based method, sample-path optimization, to solve SVIP, and Robinson [11] has provided a mathematical justification for sample-path optimization, while Shapiro et al. [12] suggested Monte Carlo sampling methods for SVIP. SVIP can be found in pricing game [13] and inventory competition [14] among several firms that provide substitutable goods or services. Some stochastic dynamic noncooperative games [15] and competitive Markov decision processes [16] can be formulated as examples of SVIP. Jiang and Xu [17] proposed a stochastic approximation method for numerical solution of SVIP. The 
method is an iterative scheme where, at each iterate, a correction is made and the correction is obtained by sampling or other stochastic approximation. Xu [18] applied the well-known sample average approximation (SAA, for short) method to solve the same class of stochastic variational inequality problems (SVIP).

Aimed at a practical treatment of the SVIP, box-constrained stochastic variational inequality problem (BSVIP, for short) is meaningful and interesting to study [19]. Motivated by Sun and Womersley [8], Luo and Lin [20] formulated a class of BSVIP as an optimization problem that minimizes the expected residual of the merit function derived on the Fishcher-Burmeister function and proposed a Monte Carlo sampling method for solving the problem.

In this paper, we consider the following $\operatorname{BSVIP}(F, S)$ to find a vector $x^{*} \in S$ such that

$$
\left(x-x^{*}\right)^{T} \mathbb{E}\left[F\left(x^{*}, \xi(\omega)\right)\right] \geq 0, \quad \forall x \in S \omega \in \Omega \text { a.s. }
$$

where $\xi: \Omega \longrightarrow \Xi \subset \mathbb{R}^{m}$ is a random vector defined on probability space $(\Omega, \mathscr{F}, P), F: S \times \mathbb{R}^{m} \longrightarrow \mathbb{R}^{n}$ is a mapping, $\mathbb{E}$ denotes the mathematical expectation with respect to the distribution of $\xi(\omega)$, and "a.s." is the abbreviation for "almost surely" under the given probability measure. To ease the notation, we will write $\xi(\omega)$ as $\xi$, and this should be distinguished from $\xi$ being a deterministic vectors of $\Xi$ in the context.

We are concerned with the numerical solution of BSVIP. If we are able to obtain a closed form of $\mathbb{E}\left[F\left(x^{*}, \xi\right)\right]$, then BSVIP becomes a deterministic BVIP, and the existing numerical methods for the latter [8] can be applied directly. However, in practice, obtaining a closed form of $\mathbb{E}\left[F\left(x^{*}, \xi\right)\right]$ or computing the value of it numerically is often difficult either due to the unavailability of the distribution of $\xi$ or because it involves multiple integration.

Motivated by the above work, we make use of a merit function derived from the Fishcher-Burmeister function (FB-function, for short) and formulate BSVIP as an optimization problem that minimizes this merit function. We study a sufficient condition for the existence of a solution to the optimization problem. Finally, we propose a Monte Carlo sampling method for solving the problem. Under some moderate conditions, comprehensive convergence analysis is included as well.

The organization of this paper is as follows. In Section 2, we study some preliminary knowledge. Section 3 shows under what conditions the level sets of the merit function are bounded. In Section 4, we make use of a Monte Carlo sampling method to handle the expectation; moreover, we establish convergence of global optimal solutions of approximation problems generated by the proposed method. Preliminary numerical results are reported in Section 5. Finally, we give some conclusions.

\section{Preliminaries}

We first consider a merit function derived from the NCPfunction [21] for BVIP (1). It is easy to see that BVIP is equivalent to its Karush-Kuhn-Tucker (KKT, for short) system:

$$
\begin{aligned}
& F(x)+\sum_{u_{i} \neq+\infty} \lambda_{i} e^{i}-\sum_{l_{j} \neq-\infty} \mu_{i} e^{j}=0, \\
& 0 \leq \lambda_{i} \perp x_{i}-u_{i} \leq 0, \quad u_{i} \neq+\infty, i=1, \ldots, n, \\
& 0 \leq \mu_{j} \perp x_{j}-l_{j} \geq 0, \quad l_{j} \neq-\infty, j=1, \ldots, n,
\end{aligned}
$$

where $e^{i}$ is a $n$-dimensional unit vector. If $x \in R^{n}$ solves BVIP, then $(x, \lambda, \mu) \in R^{n} \times R^{n} \times R^{n}$ solve the KKT system (3). Conversely, if $(x, \lambda, \mu) \in R^{n} \times R^{n} \times R^{n}$ solve the KKT system (3), then $x$ solves BVIP (1).

Let $\phi$ be a NCP-function. Then, the KKT system (3) can be written as

(i) If $l_{i}=-\infty, u_{i}=+\infty, 1 \leq i \leq n$, then $F_{i}(x)=0$.

(ii) If $l_{i} \neq-\infty, u_{i}=+\infty, 1 \leq i \leq n$, then $\mu_{i}=F_{i}(x)$, $0 \leq F_{i}(x) \perp x_{i}-l_{i} \geq 0$, and we have

$$
\phi\left(F_{i}(x), x_{i}-l_{i}\right)=0 \text {. }
$$

(iii) If $l_{i}=-\infty, u_{i} \neq+\infty, 1 \leq i \leq n$, then $\lambda_{i}=-F_{i}(x)$, $0 \geq F_{i}(x) \perp x_{i}-u_{i} \leq 0$, and we have

$$
\phi\left(-F_{i}(x), u_{i}-x_{i}\right)=0 \text {. }
$$

(iv) If $l_{i}<x_{i}<u_{i}, 1 \leq i \leq n$, then

$$
\left\{\begin{array} { l } 
{ F _ { i } ( x ) + \lambda _ { i } - \mu _ { i } = 0 , } \\
{ 0 \leq \lambda _ { i } \perp x _ { i } - u _ { i } \leq 0 , } \\
{ 0 \leq \mu _ { i } \perp x _ { i } - l _ { i } \geq 0 , }
\end{array} \Leftrightarrow \left\{\begin{array}{l}
0 \leq \lambda_{i} \perp x_{i}-u_{i} \leq 0 \\
0 \leq F_{i}(x)+\lambda_{i} \perp x_{i}-l_{i} \geq 0
\end{array}\right.\right.
$$

and we have

$$
\left\{\begin{array}{l}
\phi\left(\lambda_{i}, u_{i}-x_{i}\right)=0, \\
\phi\left(F_{i}(x)+\lambda_{i}, x_{i}-l_{i}\right)=0,
\end{array} \Leftrightarrow\left|\phi\left(\lambda_{i}, u_{i}-x_{i}\right)\right|+\left|\phi\left(F_{i}(x)+\lambda_{i}, x_{i}-l_{i}\right)\right|=0\right.
$$

Based on $\phi$, we let $\Phi_{i}(x)$ as 


$$
\Phi_{i}(x):= \begin{cases}F_{i}(x, \xi), & \text { if } l_{i}=-\infty, u_{i}=+\infty, \\ \phi\left(F_{i}(x, \xi), x_{i}-l_{i}\right), & \text { if } l_{i} \neq-\infty, u_{i}=+\infty, \\ \phi\left(-F_{i}(x, \xi), u_{i}-x_{i}\right), & \text { if } l_{i}=-\infty, u_{i} \neq+\infty, \\ \left|\phi\left(\lambda_{i}, u_{i}-x_{i}\right)\right|+\left|\phi\left(F_{i}(x)+\lambda_{i}, x_{i}-l_{i}\right)\right|, & \text { if } l_{i} \neq-\infty, u_{i} \neq+\infty .\end{cases}
$$

Then, it is easy to see that

$$
x^{*} \text { solves BVIP } \Leftrightarrow x^{*} \text { solves } \Phi(x)=0 .
$$

In turn, corresponding to this reformulation of the BVIP, we may define the merit function:

$$
\Psi(x):=\frac{1}{2} \Phi(x)^{T} \Phi(x),
$$

Therefore, $\Psi(x)$ is an unconstrained differentiable merit function for BVIP (1). In order to define this merit function, we may consider the FB-function, which is given by

$$
\phi(a, b)=(a+b)-\sqrt{a^{2}+b^{2}},
$$

and let us introduce a partition of the index set $I$ :

$$
\begin{aligned}
I_{f} & :=\left\{i \in I \mid-\infty=l_{i}<u_{i}=+\infty\right\}, \\
I_{l} & :=\left\{i \in I \mid-\infty<l_{i}<u_{i}=+\infty\right\}, \\
I_{u} & :=\left\{i \in I \mid-\infty=l_{i}<u_{i}<+\infty\right\}, \\
I_{F_{+}} & :=\left\{i \in I \mid-\infty<l_{i}<u_{i}<+\infty, \mathbb{E}\left[F_{i}(x, \xi)\right] \geq 0\right\}, \\
I_{F_{-}} & :=\left\{i \in I \mid-\infty<l_{i}<u_{i}<+\infty, \mathbb{E}\left[F_{i}(x, \xi)\right]<0\right\} .
\end{aligned}
$$

Consequently, we give the merit function for BSVIP (2) as follows:

$$
\theta(x)=\frac{1}{2}\left\{\sum_{i \in I_{f}}\left(\mathbb{E}\left[F_{i}(x, \xi)\right]\right)^{2}+\sum_{i \in I_{l} \cup I_{F_{+}}} \phi^{2}\left(\mathbb{E}\left[F_{i}(x, \xi)\right], x_{i}-l_{i}\right)+\sum_{i \in I_{u} \cup I_{F_{-}}} \phi^{2}\left(-\mathbb{E}\left[F_{i}(x, \xi)\right], u_{i}-x_{i}\right)\right\}
$$

That is to say, we formulate BSVIP as the following optimization problem:

$$
\begin{aligned}
& \min , \theta(x), \\
& \text { s.t., } x \in S .
\end{aligned}
$$

Throughout, we assume that $F(x, \xi)$ is continuous with respect to $x$ for any $\xi \in \Xi$. Furthermore, suppose that the sample space $\Xi$ is nonempty, and for every $x \in S$,

$$
\begin{aligned}
& \mathbb{E}\left[\|F(x, \xi)\|^{2}\right]<+\infty, \\
& \mathbb{E}\left[\left\|\nabla_{x} F(x, \xi)\right\|\right]<+\infty, \\
& \mathbb{E}\left[\|F(x, \xi)\|\left\|\nabla_{x} F(x, \xi)\right\|\right]<+\infty,
\end{aligned}
$$

where $\|\cdot\|$ means the Euclidean norm. By the above assumption and Th16.8 in [22], we get that $f(x):=\mathbb{E}[F(x, \xi)]$ is continuous with respect to $x$.

\section{Boundedness of Level Sets}

In this section, we discuss conditions for boundedness of the level sets of the merit function (13). Consider the level set defined by

$$
L_{\theta}^{S}(c):=\{x \in S \mid \theta(x) \leq c\}
$$

where $c \geq 0$ is a given scalar.
Definition 1. A function $f: \mathbb{R}^{n} \longrightarrow \mathbb{R}$ is a uniform $P$-function if there exists a positive constant $\mu$ such that, for every $x$ and $y$ in $\mathbb{R}^{n}$,

$$
\max _{1 \leq i \leq n}\left(x_{i}-y_{i}\right)\left(f_{i}(x)-f_{i}(y)\right) \geq \mu\|x-y\|^{2} .
$$

Now let us focus on the properties of FB-function $\phi(a, b)$.

Lemma 1. For given $a, b \in R$, we have $F B$-function $\phi(a, b)$ satisfying

$$
\frac{2}{3+2 \sqrt{2}} \min ^{2}(a, b) \leq \phi^{2}(a, b) \leq(6+4 \sqrt{2}) \min ^{2}(a, b)
$$

Proof. From Tseng [23], for any two numbers $a, b \in R$, we have

$$
\frac{2}{2+\sqrt{2}}|\min (a, b)| \leq|\phi(a, b)| \leq(2+\sqrt{2})|\min (a, b)| .
$$

Then,

$\frac{2}{3+2 \sqrt{2}} \min ^{2}(a, b) \leq \phi^{2}(a, b) \leq(6+4 \sqrt{2}) \min ^{2}(a, b)$.

Then, we study the boundedness of the level sets. 
Theorem 1. Suppose that $f(x):=\mathbb{E}[F(x, \xi)]$ is a uniform $P$-function. Then, for any $c \geq 0, L_{\theta}^{S}(c)$ is bounded.

Proof. Suppose that there is a nonnegative number $\bar{c}$ such that $L_{\theta}^{S}(\bar{c})$ is unbounded. This implies that there exists a sequence $x^{k} \subseteq L_{\theta}^{S}(c)$ such that $\lim _{k \longrightarrow \infty}\left\|x^{k}\right\|=+\infty$. We first define the index set $I \subseteq\{1, \ldots, n\}$ by

$$
I:=\left\{i \mid \lim _{k \longrightarrow \infty}\left\|x_{i}^{k}\right\|=+\infty\right\} .
$$

By assumption, we have $I \neq \varnothing$. We now define a new sequence $\left\{y^{k}\right\} \subseteq \mathbb{R}^{n}$ as follows:

$$
y_{i}^{k}:= \begin{cases}0, & \text { if } i \in I, \\ x_{i}^{k}, & \text { if } i \notin I .\end{cases}
$$

From the definition of $y^{k}$ and the fact that $f$ is a uniform $P$-function, we obtain that, for some constant $\mu>0$,

$$
\begin{aligned}
\mu \sum_{i \in I}\left(x_{i}^{k}\right)^{2} & =\mu\left\|x^{k}-y^{k}\right\|^{2} \leq \max _{1 \leq i \leq n}\left(x_{i}^{k}-y_{i}^{k}\right)\left(f_{i}\left(x^{k}\right)-f_{i}\left(y^{k}\right)\right) \\
& =\max _{i \in I} x_{i}^{k}\left(f_{i}\left(x^{k}\right)-f_{i}\left(y^{k}\right)\right) \leq \sqrt{\sum_{i \in I}\left(x_{i}^{k}\right)^{2}} \sum_{i \in I}\left|f_{i}\left(x^{k}\right)-f_{i}\left(y^{k}\right)\right|,
\end{aligned}
$$

from which we obtain

$$
\mu \sqrt{\sum_{i \in I}\left(x_{i}^{k}\right)^{2}} \leq \sum_{i \in I}\left|f_{i}\left(x^{k}\right)-f_{i}\left(y^{k}\right)\right| .
$$

By definition, the sequence $y^{k}$ remains bounded. From the continuity of $f$, it follows that the sequence $\left\{f_{i}\left(y^{k}\right)\right\}$ is also bounded for every $i=1, \ldots, n$. Hence, we deduce from (24) that there is at least one index $i_{0} \in I$ such that

$$
\begin{aligned}
x_{i_{0}}^{k} & \longrightarrow \infty, \\
f_{i_{0}}\left(x^{k}\right) & \longrightarrow \infty, \quad k \longrightarrow+\infty .
\end{aligned}
$$

In what follows, we will show that

$$
\begin{aligned}
\theta\left(x^{k}\right)= & \frac{1}{2}\left\{\sum_{i \in I_{f}}\left(\mathbb{E}\left[F_{i}\left(x^{k}, \xi\right)\right]\right)^{2}+\sum_{i \in I_{l} \cup I_{F_{+}}} \phi^{2}\left(\left(\mathbb{E}\left[F_{i}\left(x^{k}, \xi\right)\right]\right), x_{i}-l_{i}\right)+\sum_{i \in I_{u} \cup I_{F_{-}}} \phi^{2}\left(-\left(\mathbb{E}\left[F_{i}\left(x^{k}, \xi\right)\right]\right), u_{i}-x_{i}\right)\right\} \\
& \longrightarrow+\infty, \quad k \longrightarrow+\infty
\end{aligned}
$$

Now, we consider above three cases, respectively.

Case $1\left(i \in I_{f}\right): \quad$ obviously, $\quad\left(\mathbb{E}\left[F_{i_{0}}\left(x^{k}, \xi\right)\right]\right)^{2}=f_{i_{0}}^{2}$ $\left(x^{k}\right) \longrightarrow+\infty, k \longrightarrow+\infty$.

Case $2\left(i \in I_{l} \cup I_{F_{+}}\right)$: by Lemma 1, we have

$$
\begin{aligned}
& \phi^{2}\left(\mathbb{E}\left[F_{i_{0}}\left(x^{k}, \xi\right)\right], x_{i_{0}}^{k}-l_{i_{0}}\right) \\
& \geq \frac{2}{3+2 \sqrt{2}} \min ^{2}\left(\mathbb{E}\left[F_{i_{0}}\left(x^{k}, \xi\right)\right], x_{i_{0}}^{k}-l_{i_{0}}\right) .
\end{aligned}
$$

$$
\begin{aligned}
\phi^{2}\left(\mathbb{E}\left[F_{i_{0}}\left(x^{k}, \xi\right)\right], x_{i_{0}}^{k}-l_{i_{0}}\right) & \geq \frac{2}{3+2 \sqrt{2}}\left(\mathbb{E}\left[F_{i_{0}}\left(x^{k}, \xi\right)\right]\right)^{2}, \\
& =\frac{2}{3+2 \sqrt{2}} f_{i_{0}}^{2}\left(x^{k}\right) \longrightarrow+\infty, \quad k \longrightarrow+\infty
\end{aligned}
$$


Case 4: if $\min \left(\mathbb{E}\left[F_{i_{0}}\left(x^{k}, \xi\right)\right], \xi\right), x_{i_{0}}^{k}-l_{i_{0}}=x_{i_{0}}^{k}-l_{i_{0}}$, then, by (25), we have

$$
\begin{gathered}
\phi^{2}\left(\mathbb{E}\left[F_{i_{0}}\left(x^{k}, \xi\right)\right], x_{i_{0}}^{k}-l_{i_{0}}\right) \geq \frac{2}{3+2 \sqrt{2}}\left(x_{i_{0}}^{k}-l_{i_{0}}\right)^{2} \\
\longrightarrow+\infty, \quad k \longrightarrow+\infty .
\end{gathered}
$$

Case $5\left(i \in I_{u} \cup I_{F_{-}}\right)$: by Lemma 1 , we have

$$
\begin{aligned}
& \phi^{2}\left(-\mathbb{E}\left[F_{i_{0}}\left(x^{k}, \xi\right)\right], u_{i_{0}}-x_{i_{0}}^{k}\right) \\
& \geq \frac{2}{3+2 \sqrt{2}} \min ^{2}\left(-\mathbb{E}\left[F_{i_{0}}\left(x^{k}, \xi\right)\right], u_{i_{0}}-x_{i_{0}}^{k}\right) .
\end{aligned}
$$

Case $6:$ if $\min \left(-\mathbb{E}\left[F_{i_{0}}\left(x^{k}, \xi\right)\right], u_{i_{0}}-x_{i_{0}}^{k}\right)=-\mathbb{E}\left[F_{i_{0}}\left(x^{k}\right.\right.$, $\xi)]$, it follows from (25) that

$$
\begin{aligned}
{\left[\phi^{2}\left(-\mathbb{E}\left[F_{i_{0}}\left(x^{k}, \xi\right)\right], u_{i_{0}}-x_{i_{0}}^{k}\right)\right] } & \geq \frac{2}{3+2 \sqrt{2}}\left(\mathbb{E}\left[F_{i_{0}}\left(x^{k}, \xi\right)\right]\right)^{2}, \\
& =\frac{2}{3+2 \sqrt{2}} f_{i_{0}}^{2}\left(x^{k}\right) \longrightarrow+\infty, \quad k \longrightarrow+\infty .
\end{aligned}
$$

Case 7: if $\min \left(-\mathbb{E}\left[F_{i_{0}}\left(x^{k}, \xi\right)\right], u_{i_{0}}-x_{i_{0}}^{k}\right)=u_{i_{0}}-x_{i_{0}}^{k}$, then, by (25), we have

$$
\begin{aligned}
& \phi^{2}\left(-\mathbb{E}\left[F_{i_{0}}\left(x^{k}, \xi\right)\right], u_{i_{0}}-x_{i_{0}}^{k}\right) \geq \frac{2}{3+2 \sqrt{2}}\left(x_{i_{0}}^{k}-u_{i_{0}}\right)^{2} \\
& \longrightarrow+\infty, \quad k \longrightarrow+\infty .
\end{aligned}
$$

Based on the above fact, we have

$$
\bar{c} \geq \theta\left(x^{k}\right) \longrightarrow+\infty, \quad k \longrightarrow+\infty .
$$

This is a contradiction, and hence, $L_{\theta}^{S}(c)$ is bounded for any $c \geq 0$.

Since $S$ is nonempty, we see from Theorem 1 that problem (14) has at least one optimal solution when $f$ is a uniform $P$-function on $S$.
Note that the expectation function of problem (14) is generally difficult to evaluate exactly. In what follows, we employ a Monte Carlo sampling method for numerical integration to address this question.

\section{Monte Carlo Sampling Method and Convergence Analysis}

We can view the generated sample $\xi^{1}, \xi^{2}, \ldots$, as a sequence of random vectors, each having the same probability distribution as $\xi$. If the generated random vectors are stochastically independent of each other, we say that the sample is independent identically distributed (iid). With the generated sample $\xi^{i} \in \Xi, i=1,2, \ldots, N$, we associate the sample average function:

$$
\widehat{\theta}^{N}(x):=\frac{1}{2}\left\{\sum_{i \in I_{f}}\left(\hat{f}_{i}^{N}(x)\right)^{2}+\sum_{i \in I_{l} \cup I_{F_{+}}} \phi^{2}\left(\hat{f}_{i}^{N}(x), x_{i}-l_{i}\right)+\sum_{i \in I_{u} \cup I_{F_{-}}} \phi^{2}\left(-\widehat{f}_{i}^{N}(x), u_{i}-x_{i}\right)\right\}, \quad \forall x \in S
$$

where $\hat{f}_{i}^{N}(x):=(1 / N) \sum_{j=1}^{N} F_{i}\left(x, \xi^{j}\right)$, and consider the following approximation problem of (14):

$$
\begin{aligned}
& \min , \widehat{\theta}_{N}(x), \\
& \text { s.t., } x \in S .
\end{aligned}
$$

We refer (14) as the true problem and (35) as the Monte Carlo sampling average approximation problem.

If the sample is iid, then the law of large numbers (LLN) holds pointwise. We say that the LLN holds, for $\widehat{f}^{N}(x)$, pointwise if $\widehat{f}^{N}(x)$ converges w.p.1 to $f(x)$, as $N \longrightarrow \infty$, for any fixed $x \in S$. See $[12,24,25]$, for more details about the Monte Carlo sampling method.

Theorem 2. Suppose $F(x, \xi)$ is continuous with respect to $x$ for any $\xi \in \Xi$ and $\mathbb{E}\left[\|F(x, \xi)\|^{2}\right]<+\infty, \forall x \in S$; if $x_{N}$ solves problem (35) for each $N$ and $x^{*}$ is an accumulation point of $\left\{x_{N}\right\}$ as $N$ tends to infinity, then $x^{*}$ is an optimal solution of the true problem (14) with probability one.

Proof. Let $\mathscr{X} \subseteq S$ be a compact set which w.p.1 contains a neighborhood of $x^{*}$. We first show that $\widehat{\theta}^{N}(x)$ converges 
w.p.1 to $\theta(x)$ for any $x \in \mathscr{X}$. Recall (13) and (34), and we have

$$
\begin{aligned}
\left|\widehat{\theta}^{N}(x)-\theta(x)\right|= & \frac{1}{2} \mid \sum_{i \in I_{l} \cup I_{F_{+}}}\left(\phi^{2}\left(\hat{f}_{i}^{N}(x), x_{i}-l_{i}\right)-\phi^{2}\left(f_{i}(x), x_{i}-l_{i}\right)\right)+\sum_{i \in I_{u} \cup I_{F_{-}}}\left(\phi^{2}\left(-\widehat{f}_{i}^{N}(x), u_{i}-x_{i}\right)-\phi^{2}\left(-f_{i}(x), u_{i}-x_{i}\right)\right) \\
& +\sum_{i \in I_{f}}\left(\left(\hat{f}_{i}^{N}(x)\right)^{2}-\left(f_{i}(x)\right)^{2}\right) \mid .
\end{aligned}
$$

Note that

$$
\begin{aligned}
& \left|\sum_{i \in I_{l} \cup I_{F_{+}}}\left(\phi^{2}\left(\hat{f}_{i}^{N}(x), x_{i}-l_{i}\right)-\phi^{2}\left(f_{i}(x), x_{i}-l_{i}\right)\right)\right| \\
& =\left|\sum_{i \in I_{l} \cup I_{F_{+}}}\left(\phi\left(\hat{f}_{i}^{N}(x), x_{i}-l_{i}\right)-\phi\left(f_{i}(x), x_{i}-l_{i}\right)\right)\left(\phi\left(\hat{f}_{i}^{N}(x), x_{i}-l_{i}\right)+\phi\left(f_{i}(x), x_{i}-l_{i}\right)\right)\right| \\
& =\left|\sum_{i \in I_{l} \cup I_{F_{+}}}\left(\hat{f}_{i}^{N}(x)-f_{i}(x)\right)\left(1+\frac{\hat{f}_{i}^{N}(x)+f_{i}(x)}{\sqrt{\left(\hat{f}_{i}^{N}(x)\right)^{2}+\left(x_{i}-l_{i}\right)^{2}}+\sqrt{\left(f_{i}(x)\right)^{2}+\left(x_{i}-l_{i}\right)^{2}}}\right)\left(\phi\left(\hat{f}_{i}^{N}(x), x_{i}-l_{i}\right)+\phi\left(f_{i}(x), x_{i}-l_{i}\right)\right)\right| .
\end{aligned}
$$

Since $\mathscr{X}$ is a compact set and $F(x, \xi)$ is continuous with respect to $x$ for any $\xi \in \Xi$, hence, for every $x \in \mathscr{X}$, there exists a constant $0<M_{1}<+\infty$ such that

$$
\left|\left(1+\frac{\hat{f}_{i}^{N}(x)+f_{i}(x)}{\sqrt{\left(\hat{f}_{i}^{N}(x)\right)^{2}+\left(x_{i}-l_{i}\right)^{2}}+\sqrt{\left(f_{i}(x)\right)^{2}+\left(x_{i}-l_{i}\right)^{2}}}\right)\left(\phi\left(\widehat{f}_{i}^{N}(x), x_{i}-l_{i}\right)+\phi\left(f_{i}(x), x_{i}-l_{i}\right)\right)\right| \leq M_{1}, \quad i \in I_{l} \cup I_{F_{+}} .
$$

Combining (37) and (38), we have 


$$
\begin{aligned}
& \left|\sum_{i \in I_{l} \cup I_{F_{+}}}\left(\phi^{2}\left(\hat{f}_{i}^{N}(x), x_{i}-l_{i}\right)-\phi^{2}\left(f_{i}(x), x_{i}-l_{i}\right)\right)\right| \\
& \leq M_{1} \sum_{i \in I_{l} \cup I_{F_{+}}}\left(\hat{f}_{i}^{N}(x)-f_{i}(x)\right) .
\end{aligned}
$$

Similarly, we can have that, for every $x \in \mathscr{X}$, there exists constant $0<M_{2}<+\infty$ and $0<M_{3}<+\infty$ such that

$$
\begin{gathered}
\left|\sum_{i \in I_{u} \cup I_{F_{-}}}\left(\phi^{2}\left(-\widehat{f}_{i}^{N}(x), u_{i}-x_{i}\right)-\phi^{2}\left(-f_{i}(x), u_{i}-x_{i}\right)\right)\right| \leq M_{2} \sum_{i \in I_{u} \cup I_{F_{-}}}\left(\hat{f}_{i}^{N}(x)-f_{i}(x)\right), \\
\left|\sum_{i \in I_{f}}\left(\left(\hat{f}_{i}^{N}(x)\right)^{2}-\left(f_{i}(x)\right)^{2}\right)\right| \leq M_{3} \sum_{i \in I_{f}}\left(\hat{f}_{i}^{N}(x)-f_{i}(x)\right) .
\end{gathered}
$$

Let $M=\max \left(M_{1}, M_{2}, M_{3}\right)$; by (39), (40), and (41), then

$$
\left|\hat{\theta}^{N}(x)-\theta(x)\right| \leq \frac{M}{2} \sum_{i=1}^{n}\left(\hat{f}_{i}^{N}(x)-f_{i}(x)\right) \text {. }
$$

$\widehat{f}^{N}(x)$ converges w.p. 1 to $f(x)$, as $N \longrightarrow \infty$, for any fixed $x \in S$; then, for any $(2 \varepsilon / 3 M)>0$, we have that w.p.1 for $N$ is large enough:

$$
\left|\sum_{i=1}^{n}\left(\widehat{f}_{i}^{N}(x)-f_{i}(x)\right)\right| \leq \frac{2 \varepsilon}{3 M}, \quad w \cdot \text {.p. } 1 .
$$

Obviously, for every $x \in \mathscr{X}$, we can have

$$
\left|\hat{\theta}_{N}(x)-\theta(x)\right| \leq \frac{\varepsilon}{3}, \quad w . p .1 .
$$

For $x^{*}, x_{N} \in \mathscr{X}$, we have from (44) that

$$
\begin{aligned}
& \left|\hat{\theta}_{N}\left(x^{*}\right)-\theta\left(x^{*}\right)\right| \leq \frac{\varepsilon}{3}, \quad w . p .1, \\
& \left|\hat{\theta}_{N}\left(x_{N}\right)-\theta\left(x_{N}\right)\right| \leq \frac{\varepsilon}{3}, \quad w . p .1 .
\end{aligned}
$$

Since $x_{N} \longrightarrow x^{*}$ as $N \longrightarrow \infty$ and $\widehat{\theta}_{N}(x)$ is continuous, we have

$$
\left|\widehat{\theta}_{N}\left(x_{N}\right)-\widehat{\theta}_{N}\left(x^{*}\right)\right| \leq \frac{\varepsilon}{3} .
$$

Combining (47) and (45), then there exists $N(\omega)>0$ such that w.p.1 for $N>N(\omega)$ :

$$
\begin{aligned}
\left|\hat{\theta}_{N}\left(x_{N}\right)-\theta\left(x^{*}\right)\right| \leq & \left|\widehat{\theta}_{N}\left(x_{N}\right)-\widehat{\theta}_{N}\left(x^{*}\right)\right| \\
& +\left|\hat{\theta}_{N}\left(x^{*}\right)-\theta\left(x^{*}\right)\right| \leq \frac{2 \varepsilon}{3} .
\end{aligned}
$$

Note that

$$
\left|\theta\left(x_{N}\right)-\theta\left(x^{*}\right)\right| \leq\left|\theta\left(x_{N}\right)-\widehat{\theta}_{N}\left(x_{N}\right)\right|+\left|\hat{\theta}_{N}\left(x_{N}\right)-\theta\left(x^{*}\right)\right| .
$$

Combining (46) and (48), we have that w.p.1 for sufficiently large $N$ :

$$
\left|\theta\left(x_{N}\right)-\theta\left(x^{*}\right)\right| \leq \frac{\varepsilon}{3}+\frac{2 \varepsilon}{3}=\varepsilon .
$$

This implies that w.p.1 a global minimizer of (35) becomes a global minimizer of (14); hence, it is concluded.

Section 5 will demonstrate the proposed approach.

\section{Numerical Results}

In this section, we used the notation and example in Wang et al. [26] to illustrate the model of BSVIP and the formulation. In our experiments, we used the command random in Matlab R2010a to generate pseudorandom sequences and employed fmincon to solve problem (35).

Example 1 (see [26]). Consider the stochastic variational inequality problem (2), in which $\xi$ is uniformly distributed on $\quad \Xi=[0,1], S=[0,4] \times[0,4] \times[0,4], \quad$ and $F: \mathbb{R}^{3} \times \Xi \longrightarrow \mathbb{R}^{3}$ is given by

$$
F(x, \xi)=\left(\begin{array}{c}
x_{1}-\xi x_{2}+3-2 \xi \\
-\xi x_{1}+2 x_{2}+\xi x_{3}-2-\xi \\
\xi x_{2}+3 x_{3}-3-\xi
\end{array}\right) .
$$

Namely, $l_{i}=0$ and $u_{i}=4, i=1,2,3$. This problem has a solution $x^{*}=(0,1,1)^{T}$ for each $\xi \in \Xi$. The numerical results are shown in Table 1 .

Example 2 (see [26]). Consider the stochastic variational inequality problem (2), in which $\xi$ is uniformly distributed on $\Xi=[0,1], S=[0,4] \times[0,4] \times[0,4] \quad$ and $F: \mathbb{R}^{3} \times \Xi \longrightarrow \mathbb{R}^{3}$ is given by

$$
F(x, \xi)=\left(\begin{array}{c}
x_{1}^{2}-\xi x_{2}+3-2 \xi \\
-\xi x_{1}+2 x_{2}^{2}+\xi x_{3}-2-\xi \\
\xi x_{2}+3 x_{3}^{2}-3-\xi
\end{array}\right) .
$$

Namely, $l_{i}=0$ and $u_{i}=4, i=1,2,3$. It is easy to prove that the function $E[F(x, \xi)]$ is strongly monotonous. So, this stochastic variational inequality problem has a unique 
TABLE 1: The computational results for Example 1.

\begin{tabular}{lcc}
\hline$N$ & $x_{N}=\left(x_{N, 1}, x_{N, 2}, x_{N, 3}\right)$ & $\widehat{\theta}_{N}\left(x_{N}\right)$ \\
\hline 20 & $(1.382 e-2,1.086204,1.103972)$ & $1.0861 e-2$ \\
200 & $(7.736 e-3,0.990252,1.068744)$ & $6.2177 e-3$ \\
1000 & $(5.3081 e-4,0.996378,0.987653)$ & $3.4724 e-4$ \\
10000 & $(8.2676 e-5,1.000652,1.000933)$ & $4.5232 e-5$ \\
20000 & $(3.275 e-6,1.000083,0.999951)$ & $1.2911 e-6$ \\
\hline
\end{tabular}

TAвLE 2: The computational results for Example 2.

\begin{tabular}{lcc}
\hline$N$ & $x_{N}=\left(x_{N, 1}, x_{N, 2}, x_{N, 3}\right)$ & $\widehat{\theta}_{N}\left(x_{N}\right)$ \\
\hline 20 & $(9.0123 e-3,0.932573,0.965513)$ & $9.4742 e-3$ \\
200 & $(1.7361 e-3,1.009566,1.010213)$ & $1.2275 e-3$ \\
1000 & $(8.7204 e-5,0.995087,1.008562)$ & $7.8327 e-5$ \\
10000 & $(1.3375 e-5,1.999361,1.000375)$ & $1.0253 e-5$ \\
20000 & $(1.0017 e-6,1.000027,0.999987)$ & $1.0009 e-6$ \\
\hline
\end{tabular}

solution $x^{*}=(0,1,1)^{T}$ for each $\xi \in \Xi$. The numerical results are shown in Table 2 .

From the above analysis for Examples 1-2, our preliminary numerical results for these examples indicate that the proposed method yields a reasonable and better solution of the stochastic variational inequality problem (2).

\section{Conclusions}

In this paper, the well-known Mote Carlo sampling method is applied to solve a class of box-constrained stochastic variational inequality problems. Firstly, a merit function derived from the FB-function is used and BSVIP is formulated as an optimization problem that minimizes this merit function. A sufficient condition is suggested for the existence of a solution to the optimization problem. Finally, this paper proposes a Monte Carlo sampling method for solving the problem. Under some moderate conditions, comprehensive convergence analysis is included as well.

\section{Data Availability}

The data used to support the findings of this study are from reference [26].

\section{Conflicts of Interest}

The author declares no conflicts of interest.

\section{Acknowledgments}

This study was supported by the National Natural Science Foundation of China (Grant no. 11501476) and the Southwest Minzu University Research Startup Funds.

\section{References}

[1] F. Facchinei and J. S. Pang, Finite-imensional VariationaInequalities and Complementarity Problems, vol. II, Springer-Verlag, New York, NY, USA.
[2] R. W. Cottle, J. S. Pang, and R. E. Stone, The Linear Complementarity Problrm, Academic Press, San Diego, CA, USA, 1992.

[3] M. C. Ferris and J. S. Pang, "Engineering and economic applications of complementarity problems," SIAM Review, vol. 39, no. 4, pp. 669-713, 1997.

[4] N. Hebestreit, "Vector variational inequalities and related topics: a survey of theory and applications," Applied SetValued Analysis and Optimization, vol. 1, pp. 231-305, 2019.

[5] N. Shahzad and H. Zegeye, "Convergence theorems of common solutions for fixed point, variational inequality and equilibrium problems," Journal of Nonlinear and Variational Analysis, vol. 3, pp. 189-203, 2019.

[6] Z. Kebaili and D. Benterki, "A penalty approach for a box constrained variational inequality problem," Applications of Mathematics, vol. 63, no. 4, pp. 439-454, 2018.

[7] C. Zhang, Z. Zhu, Y. Yao, and Q. Liu, "Homotopy method for solving mathematical programs with bounded box-constrained variational inequalities," Optimization, vol. 68, pp. 2293-2312, 2019.

[8] D. Sun and R. S. Womersley, "A new unconstrained differentiable merit function for box constrained variational inequality problems and a damped Gauss--Newton method," SIAM Journal on Optimization, vol. 9, no. 2, pp. 388-413, 1999.

[9] B. Chen, X. Chen, and C. Kanzow, "A penalized FischerBurmeister NCP-function," Mathematical Programming, vol. 88, no. 1, pp. 211-216, 2000.

[10] G. Gürkan, A. Y. Ozge, and S. M. Robinson, "Sample-path solution of stochastic variational inequalities," Mathematical Programming, vol. 84, pp. 313-333, 1999.

[11] S. M. Robinson, "Analysis of sample-path optimization," Mathematics of Operations Research, vol. 21, no. 3, pp. 513528, 1996.

[12] A. Shapiro, A. Ruszczyński and A. Shapiro, Monte Carlo sampling methods," in Stochastic Programming, Handbooks in $O R$ \& $M S$, vol. 10, North-Holland Publishing Company, Amsterdam, Netherlands, 2003.

[13] F. Y. Chen, H. Yan, and L. Yao, "A newsvendor pricing game," IEEE Transactions on Systems, Man, and Cybernetics-Part A: Systems and Humans, vol. 34, no. 4, pp. 450-456, 2004.

[14] S. Mahajan and G. Van Ryzin, "Inventory competition under dynamic consumer choice," Operations Research, vol. 49, no. 5, pp. 646-657, 2001.

[15] T. Basar and G. Olsder, Dynamic Noncooperative Game Theory, SIAM, Philadelphia, PA, USA, 1999.

[16] J. Filar and K. Vrieze, Competitive Markov Decision Processes, Springer-Verlag, Berlin, Germany, 1997.

[17] H. Jiang and H. Xu, "Stochastic approximation approaches to the stochastic variational inequality problem," IEEE Transactions on Automatic Control, vol. 53, no. 6, pp. 1462-1475, 2008.

[18] H. Xu, "Sample average approximation methods for a class of stochastic variational inequality problems," Asia-Pacific Journal of Operational Research, vol. 27, no. 1, pp. 103-119, 2010.

[19] J. Jiang, X. Chen, and Z. Chen, "Quantitative analysis for a class of two-stage stochastic linear variational inequality problems," Computational Optimization and Applications, vol. 76, no. 2, pp. 431-460, 2020.

[20] M. J. Luo and G. H. Lin, "Sample average approximation method for solving a deterministic formulation for box constrained stochastic variational inequality problems," AsiaPacific Journal of Operations Research, vol. 29, pp. 1-17, 2012. 
[21] D. Sun and L. Qi, "On NCP-functions," Computational Optimization and Applications, vol. 13, no. 1/3, pp. 201-220, 1999.

[22] B. Patrick, Probability and Measure, A Wiley-Interscience Publication, John Wiley \& Sons, New York, NY, USA, 1995.

[23] P. Tseng, "Growth behavior of a class of merit functions for the nonlinear complementarity problem," Journal of Optimization Theory and Applications, vol. 89, no. 1, pp. 17-37, 1996.

[24] H. Niederreiter, Random Number Generation and QuasiMonte Carlo Methods, SIAM, Philadelphia, PA, USA, 1992.

[25] A. Shapiro, "Monte Carlo sampling approach to stochastic programming," ESAIM: Proceedings, vol. 13, pp. 65-73, 2003.

[26] M. Wang, G. Lin, Y. Gao, and M. M. Ali, "Sample average approximation method for a class of stochastic variational inequality problems," Journal of Systems Science and Complexity, vol. 24, no. 6, pp. 1143-1153, 2011. 